Portland State University

PDXScholar

\title{
Teaching Doppler Ultrasound in an Introductory Laboratory for Pre-health Students
}

Theodore Stedmark

Portland State University, stenmark@pdx.edu

Thomas Allen

Portland State University

Ralf Widenhorn

Portland State University, ralfwidenhorn@pdx.edu

Follow this and additional works at: https://pdxscholar.library.pdx.edu/phy_fac

Part of the Physics Commons

Let us know how access to this document benefits you.

Citation Details

Stenmark, T., Allen, T., \& Widenhorn, R. (2020). Teaching Doppler Ultrasound in an Introductory Laboratory for Pre-health Students. The Physics Teacher, 58(3), 206-208.

This Article is brought to you for free and open access. It has been accepted for inclusion in Physics Faculty Publications and Presentations by an authorized administrator of PDXScholar. Please contact us if we can make this document more accessible: pdxscholar@pdx.edu. 


\section{Teaching Doppler Ultrasound in an Introductory Laboratory for Pre-health Students}

Cite as: Phys. Teach. 58, 206 (2020); https://doi.org/10.1119/1.5145419

Published Online: 19 February 2020

Theodore Stenmark, Thomas Allen, and Ralf Widenhorn

ARTICLES YOU MAY BE INTERESTED IN

Breathtaking Physics: Human Respiration as a Heat Engine

The Physics Teacher 58, 150 (2020); https://doi.org/10.1119/1.5145400

Helmholtz Resonator in Laboratory Experiments

The Physics Teacher 58, 179 (2020); https://doi.org/10.1119/1.5145410

Rainbows: A Graphical Approach

The Physics Teacher 58, 152 (2020); https://doi.org/10.1119/1.5145401

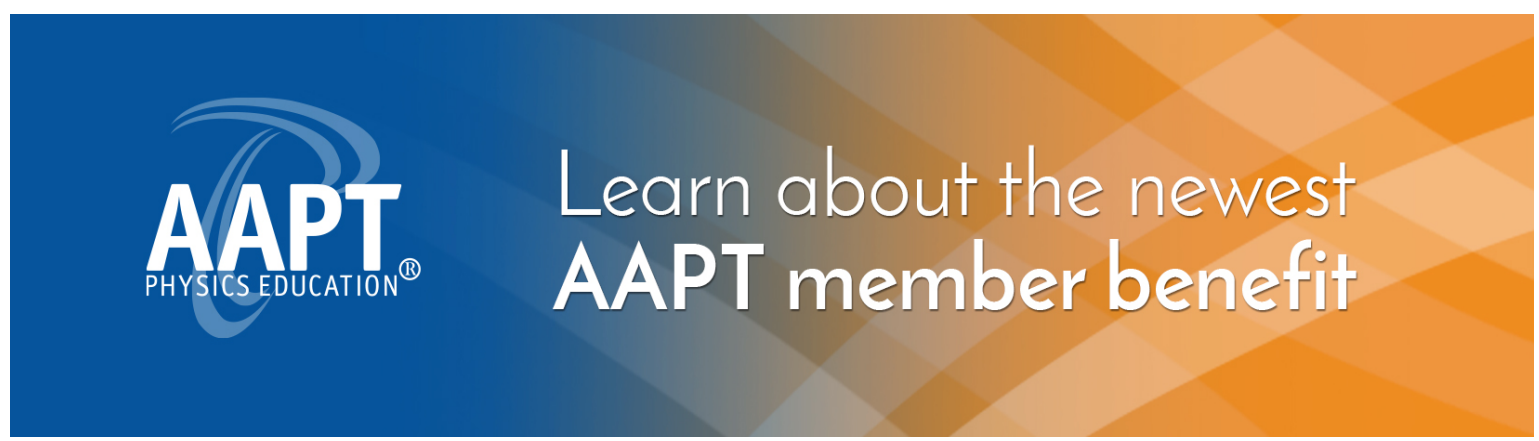

Phys. Teach. 58, 206 (2020); https://doi.org/10.1119/1.5145419

58, 206

(c) 2020 American Association of Physics Teachers. 


\section{Teaching Doppler Ultrasound in an Introductory Laboratory for Pre-health Students}

Theodore Stenmark, Thomas Allen, and Ralf Widenhorn, Portland State University, Portland, OR

W e present a novel activity to demonstrate the Doppler shift of a sound wave, incident at an angle, upon a moving reflector. This activity is intended for use in an introductory physics laboratory focused on preparing students for the health and medical fields. The activity is designed to simulate Doppler velocity measurements from ultrasound imaging. While there have been previous qualitative discussions of blood flow measurements in the physics education literature, they were without associated laboratory activities. ${ }^{1}$ The lab can be part of a life science physics curriculum that has been identified in need of reforms to meet the needs of students and the medical and biology community. ${ }^{2-7}$

\section{Doppler shift}

At nonrelativistic speeds, the change in frequency of a sound wave emitted by a moving source and observed by a moving receiver is given by

$$
f^{\prime}=\frac{c \pm v_{\mathrm{r}}}{c \pm v_{\mathrm{s}}} f_{0}
$$

where $f^{\prime}$ is the measured frequency, $c$ is the speed of sound in the media, $v_{\mathrm{r}}$ is the speed of the receiver, $v_{\mathrm{s}}$ is the speed of the source, $f_{0}$ is the emitted frequency, and the sign of the receiver and source speeds depends on the relative direction of motion.

To mimic measuring blood flow speed with ultrasound, we use an apparatus that contains a stationary initial sound source, a moving reflector, and a stationary receiver located near the source. We can consider the final frequency measured at the receiver to be the result of two Doppler shifts. The first Doppler shift results from the moving receiver reflecting the initial sound pulse, and the second Doppler shift results from the reflected wave now being "emitted" from the moving reflector. The double shift from the reflection is equivalent to that of source and receiver approaching each other at the same speed and thus,

$$
f^{\prime}=\frac{c+v}{c-v} f_{0}
$$

since $v=v_{\mathrm{S}}=-v_{\mathrm{r}}$.

Now if the sound source and receiver are oriented at an angle $\theta$ with respect to the direction of motion of the reflector, then the Doppler shifted frequency of the wave becomes

$$
f^{\prime}=\frac{c+v \cos \theta}{c-v \cos \theta} f_{0} .
$$

$\theta$ is referred to as the insonation angle in ultrasound imaging. For Doppler ultrasound imaging it represents the angle between the sound emitted by the transducer and the direction of the blood flow.

In the limit that $v \ll c$, as is the case for blood flow measurement and for the speed of the cart in this experiment, this simplifies to

$$
\Delta f=f^{\prime}-f_{0} \approx \frac{2 v \cos \theta}{c} f_{0} .
$$

The speed of the cart can then be calculated from the Doppler shift with

$$
v=\frac{c}{\cos \theta} \times \frac{f^{\prime}-f_{0}}{2 f_{0}} .
$$

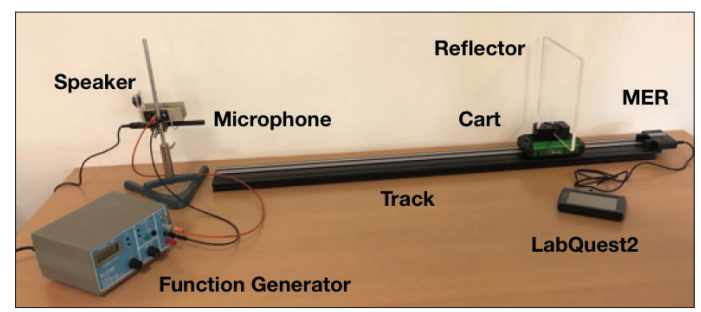

Fig. 1. Setup used in the first experiment. The microphone and speaker are mounted on the ring stand to the left of the track. The LabQuest 2 data logger/visualizer is attached to the motion encoder receiver (MER) on the right end of the track. The function generator is in front of the ring stand in this image.

\section{Apparatus}

This activity makes use of commercially available equipment and data acquisition software commonly used in introductory laboratories. The equipment includes a track with defined markings and a cart with an optical motion encoder receiver $(\mathrm{MER})^{8}$ to measure its velocity. This system is commercially available from Vernier. Additionally a custommade plastic reflector and reflector holder were machined in-house. ${ }^{9}$ The sound source is generated with a function generator and a speaker, ${ }^{10}$ and received with a Vernier MCA-BTA microphone. ${ }^{11}$ The setup is shown in Fig. 1. The LabQuest Mini and Logger Pro software were used with the microphone to acquire frequency data, while a LabQuest 2 was used with the MER to record the velocity of the cart. Alternatively, an ultrasonic motion detector or photogates could be used to measure the velocity of the cart.

The two separate interfaces were used due to the different data sampling rates needed for the sound and velocity data. If the frequency of sound used in the experiment is lowered to $\sim 4000 \mathrm{~Hz}$, both the microphone and MER may be run off of Logger Pro at a sampling rate of $\sim 10,000$ samples per second. However, this requires the experiment to be performed in the audible range, which can create a loud laboratory environment when many students are performing the experiment simultaneously. We note that the audible range is useful as an introduction to the lab or as a teaching demo using this setup.

\section{Use in the classroom}

This activity has the following objectives. First, to demonstrate to students how the Doppler shift can be used to determine the speed of an object in the specific case of a stationary sound source and receiver and a moving reflecting object. Second, to demonstrate how this model can be built upon to 
accommodate an angled, moving reflecting surface. Laboratory activities appropriate for the first objective have been discussed in the literature already (some utilize a stationary receiver and moving sound source $)^{12-17}$; however, we are not aware of a student activity that includes the angular dependence, which directly relates to real-world medical applications in ultrasound imaging.

\section{Experiment 1}

The first experiment is a measurement of the velocity of a cart moving directly toward the source using a Doppler shifted echo and comparing it with the velocity recorded by the MER. The primary factor difference of the experiment from the idealized model is variations of the cart's velocity over the data collection time. A level track will result in a decrease in velocity due to frictional forces, so a slight incline to counteract the frictional force is preferable. To achieve a constant velocity, we set the cart in motion with a light push and record the velocity. After observing the velocity vs. time graph, we tilt the track in the appropriate direction and repeat until the velocity vs. time graph becomes approximately constant. Students are asked to look at the percentage change in the velocity of the cart from one end of the track to the other and make sure it is under $5 \%$.

Once we are satisfied with the leveling of the track, a cart with an attached reflector is set into constant velocity motion along a track with a slight push; once the cart is in motion, the collect button is hit on Logger Pro with a data collection time of $1 \mathrm{~s}$. A constant, known frequency is emitted from a speaker, reflected off a panel attached to the moving cart, and recorded by a Vernier microphone at a sampling rate of $\sim 45 \mathrm{kHz}$. We use a function generator set to $20 \mathrm{kHz}$ to generate the signal. It should be noted that while the Vernier MCA-BTA Microphone is rated for only $15 \mathrm{kHz}$ and cannot cleanly define the sinusoidal waveform, the frequency can be determined from the Fourier transform. We chose $20 \mathrm{kHz}$ as it is outside the audible range, although other frequencies may be used. The sampling rate may need to be adjusted with frequency to avoid aliasing effects. Once the reflected wave is received by the microphone, it is recorded and analyzed using computer software. Here, we use the commercially available Logger Pro software to process the reflected signal with a Fourier transform, allowing the student to measure the frequency of the reflected wave.

To compare with the calculated velocity of the cart, we also directly measured the velocity of the cart with the motion encoder system with a sampling rate of 30 samples per second. The precision of the frequency can generally be obtained to within a few hertz based on the full-width, half-max (FWHM) of the Fourier transform peak. For typical cart speeds in the 0.2 to $0.6 \mathrm{~m} / \mathrm{s}$ range, the expected Doppler shift from a $20-\mathrm{kHz}$ frequency is from $\sim 25$ to $70 \mathrm{~Hz}$.

Figure 2 shows the results of one trial of this experiment. We can clearly see the emitted frequency with the function generator set to $20,000 \pm 1.5 \mathrm{~Hz}$ as well as the reflected signal with distribution peak at approximately $20,041 \pm 2 \mathrm{~Hz}$. The uncertainties for the emitted and reflected frequencies are based on the FWHM of their signal peaks in Fig. 2. The average velocity of the cart is measured to be $0.36 \mathrm{~m} / \mathrm{s}$ by the MER

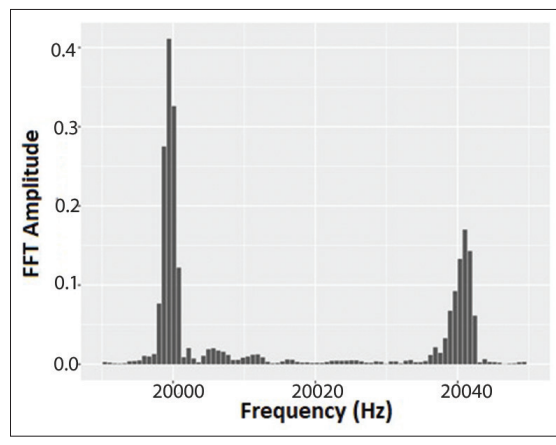

Fig. 2. Results of Experiment 1. The most prominent peak at around $20 \mathrm{kHz}$ corresponds to the frequency of the emitted frequency. The next most prominent peak at around $20.041 \mathrm{kHz}$ corresponds to the reflected peak Doppler shifted due to the motion of the cart. with a variation of $\sim 5 \%$ from one end of the track to the other. We can now calculate the velocity of the cart using the Doppler shifted frequency using the equation for $v<<c$. $v=c \times \frac{f^{\prime}-f_{0}}{2 f_{0}}$

$$
\begin{aligned}
& =343 \mathrm{~m} / \mathrm{s} \times \frac{41}{40,000} \\
& =0.35 \mathrm{~m} / \mathrm{s} .
\end{aligned}
$$

The predominant source of uncertain- ty in the velocity calculation came from the uncertainty in the magnitude of the Doppler shift, the $f^{\prime}-f_{0}$ term. We assumed the speed of sound to be known and note that the error in the $f_{0}$ term in the denominator is negligible as it is known to within $2 \mathrm{~Hz}$ out of $20 \mathrm{kHz}$. The uncertainty was estimated as follows. If $\partial f_{0}$ and $\partial f^{\prime}$ are the magnitude of uncertainty for the frequencies, then the percent uncertainty can be calculated by

$$
\text { Percent uncertainty }=\frac{\sqrt{\partial f_{0}^{2}+\partial f^{\prime 2}}}{\left|f^{\prime}-f_{0}\right|} \text {. }
$$

Using our uncertainties of $1.5 \mathrm{~Hz}$ and $2 \mathrm{~Hz}$ for $\partial f_{0}$ and $\partial f^{\prime}$, respectively, this gives us an uncertainty of $6.1 \%$, and the direct and Doppler measurements of the speed agree within the estimated uncertainty. By simply reversing the direction of motion of the cart and releveling the track, this experiment can be modified to measure the velocity of a receding object.

\section{Experiment 2}

The second experiment builds off of the first and provides a novel activity particularly suited for health science students. For this experiment the sound source, receiver, and the reflector are all placed at an angle with respect to the direction of motion of the cart. Figure 3 shows the setup for this experiment. The cart that we use has a custom-made mount that allows the reflector to be placed at angles of $15^{\circ}, 30^{\circ}$, and $45^{\circ}$ with respect to the direction of motion.

Figure 4 shows the results of one trial with this setup with an emitted frequency of $20,002 \pm 1.5 \mathrm{~Hz}$ and a Doppler shifted frequency of $20,036 \pm 1.5 \mathrm{~Hz}$. In this case the speaker and microphone are set to an angle of $30 \pm 0.5^{\circ}$ with respect to the center of the track, and the reflecting panel is adjusted to the $30^{\circ}$ slot to maximize the efficiency of the reflection. The speaker and microphone should not be placed too close to the track to reduce the variation in angle as the cart rolls along the track. A distance of 1 to $2 \mathrm{~m}$ is generally sufficient. For the data presented below, the speaker and microphone were placed at a distance of $1.5 \mathrm{~m}$. In the same manner as the first experiment, the cart is given a slight push to set it in motion. The data collection time is set to $1 \mathrm{~s}$ and some coordination is required to ensure the data collection window includes the time in which the cart passes through the center of the track. 


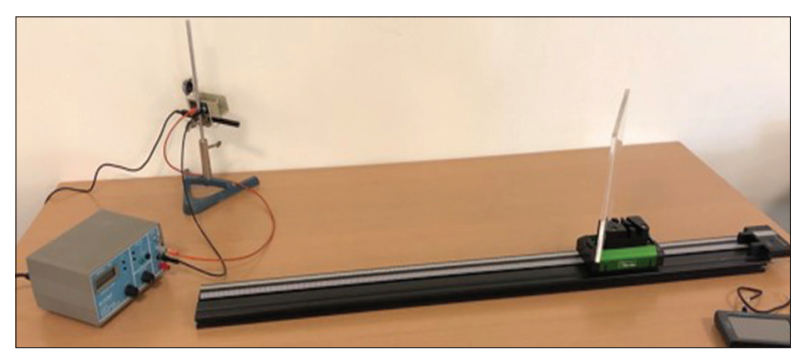

Fig. 3. Setup used in the second experiment. The setup is similar to the first experiment, except now the reflector and the sound source are at an angle with respect to the motion of the cart.

We directly measure the average velocity of the cart to be 0.35 $\mathrm{m} / \mathrm{s}$, with a variation of $\sim 5 \%$ from one end of the track to the other. We can solve the Doppler shift equation, accounting for the angle, to determine the velocity of the cart.

$$
\begin{aligned}
v & =\frac{c}{\cos \theta} \times \frac{f^{\prime}-f_{0}}{2 f_{0}} \\
& =\frac{343 \mathrm{~m} / \mathrm{s}}{\cos 30^{\circ}} \times \frac{20,036-20,002}{40,004} \\
& =0.34 \mathrm{~m} / \mathrm{s} .
\end{aligned}
$$

Students should realize there is an additional variable with uncertainty being added, namely the angle. Due to the cosine term, the error propagation for the speaker and microphone placement angle is more complex, but students can compare values for different angles. For example, over the 1-s collection time, the cart travels $35 \mathrm{~cm}$ in our sample data, which will produce a range of angles. If we assume the data collection window is centered on the time when the cart was in the center of the track where the angle is $30^{\circ}$, this corresponds to an angular change of $\pm 3^{\circ}$ when the speaker is at a distance of 1.5 $\mathrm{m}$. Students can compare $\cos \left(27^{\circ}\right)$ and $\cos \left(33^{\circ}\right)$ and find the maximum uncertainty from the changing angle to be $\sim 6 \%$. It should also be noted that the efficiency of reflection of the signal back towards the microphone is strongest when the cart is at the $30^{\circ}$ angle, which helps to mitigate this error. We also calculate the uncertainty from the Doppler shift due to the

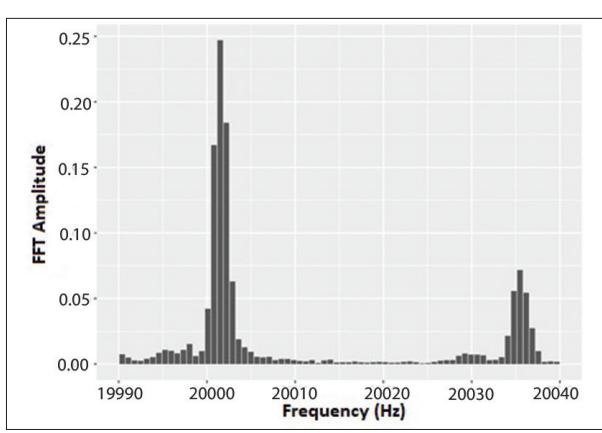

Fig. 4. Results of Experiment 2. The emitted frequency from the FFT is at $20,002 \pm 1.5 \mathrm{~Hz}$ while the reflected frequency Doppler shifted due to the motion of the cart is at $20,036 \pm$ $1.5 \mathrm{~Hz}$. typically within $\sim 5 \%$ of each other.

\section{Conclusion}

We present an experiment that expands upon a typical Doppler shift laboratory to measure the velocity of an object moving at an angle with respect to the sound source. This experiment is designed for students in health science fields as a simple model of Doppler velocity measurements used for example in blood flow measurements.

\section{References}

1. H. R. Crane, "Folded-path Doppler and the measurement of blood flow," Phys. Teach. 37, 362-363 (Sept. 1999).

2. AAPT, "Conference on Introductory Physics for the Life Sciences Report” (2014), ISBN: 978-1-931024-25-9, https://www. compadre.org/IPLS/documents/IPLS-Final-Report.pdf.

3. R. C. Hilborn and M. J. Friedlander "Biology and physics competencies for pre-health and other life science students, " $C B E$ Life Sci. Educ. 12 (2), 170 (2013).

4. D. C. Meredith and E. F. Redish, "Reinventing physics for life-science majors," Phys. Today 66 (7), 38 (2013).

5. AAAS, "Vision and Change in Undergraduate Biology Education" (American Association for the Advancement of Science, Washington, DC, 2011).

6. AAMC-HHMI, "Scientific Foundations for Future Physicians" (Association of American Medical Colleges, Washington, DC, 2009).

7. C. H. Crouch, R. Hilborn, S. A. Kane, T. McKay, and M. Reeves, "Physics for future physicians and life scientists: A moment of opportunity," APS News 19 (3), 8 (2010), http://www.aps.org/ publications/apsnews/201003/backpage.cfm.

8. Vernier Motion Encoder Receiver, https://www.vernier.com/ products/lab-equipment/dynamics/vds-ec/.

9. Custom reflector mount, https://github.com/psuphysicsedres/ Doppler-Lab.

10. Speaker Model Number: Dayton Audio CE Series CE32A-4 1-1/4" Mini Speaker 4 Ohm.

11. Vernier MCA-BTA Microphone, https://www.vernier.com/ products/sensors/microphones/mca-bta/.

12. José A. Gómez-Tejedor, Juan C. Castro-Palacio, and Juan A. Monsoriu, "The acoustic Doppler effect applied to the study of linear motions," Eur. J. Phys. 35 (2), 025006 (2014). https://doi. org/10.1088/0143-0807/35/2/025006.

13. A. Azooz, "Experimental demonstration of Doppler spectral broadening using the PC sound card," Am. J. Phys. 75, 184-188 (Feb. 2007).

14. T. J. Bensky and S. E. Frey, "Computer sound card assisted measurements of the acoustic Doppler effect for accelerated and unaccelerated sound sources," Am. J. Phys. 69, 1231-1236 (Dec. 2001).

15. A. J. Cox and J. J. Peavy, "Quantitative measurements of the acoustic Doppler effect using a walking speed source," Am. J. Phys. 66, 1123-1125 (Dec. 1998).

16. S. J. Spicklemire and M. A. Coffaro, "The treatment of reflections in a Doppler measurement using the method of images," Am. J. Phys. 74, 40-42 (Jan. 2005).

17. S. M. Torres and W. J. González-Espada, "Calculating $g$ from acoustic Doppler data," Phys. Teach. 44, 536-539 (Oct. 2006).

Portland State University, Portland, OR; stenmark@pdx.edu 